This item was submitted to Loughborough's Research Repository by the author.

Items in Figshare are protected by copyright, with all rights reserved, unless otherwise indicated.

\title{
Distributed autonomous real-time planning and control of small to medium enterprises
}

PLEASE CITE THE PUBLISHED VERSION

PUBLISHER

Professional Engineering Publishing / @ IMechE

VERSION

VoR (Version of Record)

LICENCE

CC BY-NC-ND 4.0

\section{REPOSITORY RECORD}

Rahimifard, Shahin, Stephen T. Newman, and R. Bell. 2019. "Distributed Autonomous Real-time Planning and Control of Small to Medium Enterprises". figshare. https://hdl.handle.net/2134/5059. 
This item was submitted to Loughborough's Institutional Repository (https://dspace.lboro.ac.uk/) by the author and is made available under the following Creative Commons Licence conditions.

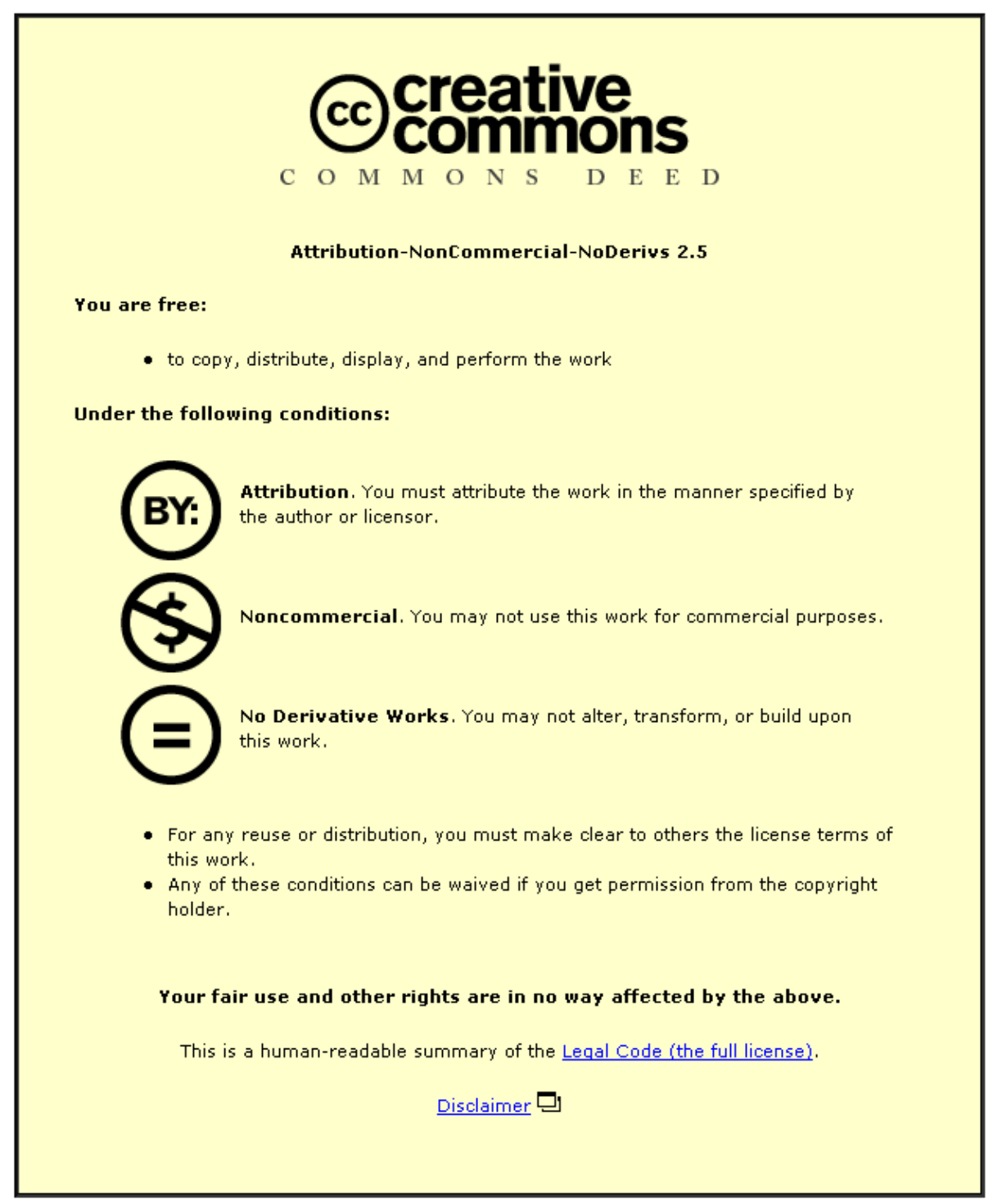

For the full text of this licence, please go to: http://creativecommons.org/licenses/by-nc-nd/2.5/ 


\title{
Distributed autonomous real-time planning and control of small to medium enterprises
}

\author{
S Rahimifard*, S T Newman and R Bell \\ Department of Manufacturing Engineering, Loughborough University, UK
}

\begin{abstract}
The research reported in this paper is built on the use of the concepts of tholonic manufacturing' and is seeking new methods to reinforce the autonomous and cooperative attributes of planning and control activities within human centred manufacturing facilities. It is claimed that in small enterprises, where the success depends on the skill of individual employees and their ability to collaborate, the manufacturing performance can be improved by the use of the appropriate information technology (IT) tools and the appropriate planning and control structure, designed and developed on the basis of these holonic concepts. Such novel tools and planning structures must provide the most appropriate information in a way that enhances collaborative activity within the business and strengthens the role of the individual. This paper identifies a number of major requirements that must be considered for the design and implementation of an effective production planning and control (PPC) structure, tailored for the needs of small enterprises. Based on these requirements and the use of holonic manufacturing concepts, a novel distributed autonomous real-time planning and control structure is presented. The final sections of the paper describe the implementation of such a planning and control structure within a typical configuration for the production facility of a small metalworking enterprise.
\end{abstract}

Keywords: real-time planning, holonic manufacturing, distributed scheduling

\section{INTRODUCTION}

In recent years, manufacturing industries have been forced to show greater flexibility in order to deal with both the increase in variety of products and reduction in batch sizes. In the 1980s it was commonly assumed that the implementation of computer integrated manufacturing (CIM) concepts would provide such flexibility. This notion is now being challenged by the increasing belief that the implementation of CIM results in manufacturing systems that are in general too rigid and that the attributes of such highly integrated systems with totally predictable patterns of operation will not meet the needs of a modern industry that has to be highly reactive. In pursuit of this greater reactivity, there is a growing opinion that current CIM-based organizations should be replaced by more innovative and organic structures. One such exemplar is a conglomerate of distributed and autonomous units operat-

The MS was received on 17 July 1998 and was accepted after revision for publication on 12 November 1998.

*Corresponding author: Department of Manufacturing Engineering, Loughborough University, Loughborough, Leicestershire LE11 3TU, UK. ing as a set of cooperating entities. In this respect, new concepts have been proposed which include holonic manufacturing [1], bionic cells [2] and fractal factories [3]. Readers are referred to Tharumarajah et al. [4] for a critical comparison of these concepts.

Small to medium enterprises (SMEs) represent the largest proportion of the manufacturing sector, generating more than half of the total production output in every industrial country $[\mathbf{5 , 6}]$. It is also commonly reported that the quality and consistency of the manufacturing planning and control of these SMEs can be improved as a consequence of the use of the most appropriate information technology (IT) tools, without a need for any major changes in business practices, manufacturing operations or the production facilities $[7,8]$. The requirements for such IT tools have intensified in recent years, as manufacturing SMEs have experienced an urgency for greater flexibility in dealing with highly individualistic customer desires for participation in design and production within the 'engineering-to-order' sector and the unpredictable pattern of demands in the 'make-to-order' sector $[\mathbf{9 , 1 0 ]}$. In addition, it is claimed that the success of small companies is very much dependent on the skill of individual operators 
and their ability to collaborate. The research reported in this paper is seeking a way to reinforce such autonomous and cooperative attributes through the use of holonic manufacturing concepts within human centred manufacturing applications. The initial part of the paper briefly reviews the most recent holonic manufacturing research and identifies the major requirements for a holonic planning and control structure specifically tailored to meet the needs of SMEs. The latter part of the paper outlines the structure for a novel distributed autonomous real-time production planning and control system developed on the basis of these requirements and describes the implementation of such a structure within a metalworking SME.

\section{REVIEW OF HOLONIC MANUFACTURING RESEARCH}

The origins of the holonic paradigm are to be found in work by Koestler [11] in the field of experimental psychology. The core of Koestler's argument was that the materialization of any highly complex system had to be established on the basis of a hierarchy composed of stable, intermediary structures on a series of levels. The intermediary organizational structures were described as assertive or self-contained wholes and, at the same time, integrative or dependent parts within a more expansive hierarchy. Koestler introduced the term 'holon' to describe nodes on such organizational structures. This word is derived from the prefix holo (from the Greek 'holos', meaning complete or whole) and the suffix on (from the Greek 'on', meaning elementary particle or part). Another concept introduced by Koestler was that of a 'holarchy', which is a system consisting of holons as building blocks; this identified a stable, complex system within which self-contained intermediate forms can be distinguished.

In general, the term 'holonic manufacturing' refers to applications of Koestler's fundamental concepts of the holon in the design and development of future manufacturing systems. Leeuwen and Norrie [12] describe the holon as an autonomous, cooperative building block of a manufacturing system for transforming, transporting, storing and validating information and physical objects. Autonomy is defined as the capability of an entity to create and control the execution of its own plans and strategies, and cooperation as the process whereby a set of interacting entities develop and execute mutually acceptable plans.

Research on holonic manufacturing sprang into prominence as part of a Japanese initiative in 1989 to explore the use of holonic systems as an effective manufacturing paradigm, which was reported by Suda [1] in the project formed by the Japanese state programme on fifth-generation computing. This work has been taken up by the international initiative on intelligent manufacturing systems (IMS) [13, 14]. This initiative on holonic manufacturing systems (HMS) now involves a multinational collaborative research activity under the IMS programme [15], consisting of a consortium of several international companies and research institutes. This international consortium includes manufacturing industries and research institutes from Australia, Canada, the European Community (EU), European Free Trade Association (EFTA), Japan and the United States. The early work concentrated on highly automated systems [16, 17], whereas, more recently, emphasis has been given to work on human centred manufacture $[18,19]$.

A diverse range of research work on holonic manufacturing can be encountered in the literature. The most relevant of these to the research reported in this paper are the use of holonic concepts in production scheduling [20-23], shop-floor control [24, 25] and developing intelligent controllers for manufacturing workstations $[\mathbf{2 6}, \mathbf{2 7}]$. In this context, a major research initiative on holonic manufacturing systems is being pursued by the Production Engineering, Machine Design and Automation (PMA) division of the University of Leuven, Belgium, within IMS test case No. 5 and a nationally funded project entitled GOA-95/2 [28]. The major research activities are directed at the definition of a reference architecture and design methodology for holonic manufacturing systems, the development and analysis of a holarchy prototype and the generation of a number of holon prototypes [29]. A part of this research initiative is aimed at the realization of a holonic architecture for a shop-floor control system. The architecture supports the use of several control strategies, such as hierarchical control, heterarchical control and other holonic control strategies. These strategies are based on tight cooperation between a scheduler and shop-floor control holons [30]. These holons are made to react autonomously to manufacturing disturbances while considering global performance. To achieve this, a suite of holarchies for process planning and resource aggregation has been developed, consisting of resource holons, order holons, product holons and staff (process planner, scheduler, shop-floor controller) holons. The research also focuses on the architecture of autonomous workstations in holonic manufacturing, which comprises resource allocation holons and process control holons [31].

In addition, another relevant programme of complementary work is the research initiatives on holonic manufacturing within the European Eureka/Factory project, REMAPHOS (EU-1584), which are aimed at the development of a generic methodology for the restructuring of large-scale manufacturing units into autonomous work units [32]. A major emphasis of this project is to develop a computer assisted management system to support a broad analysis of company 
facilities, products, procedures and human resources to launch autonomous business units. It is expected that the resultant network of businesses will resemble a holonic production system. This project includes a pilot implementation in a large-scale, state owned enterprise in the field of machine tool manufacture [33]. Initial results from the first working group meeting include a conceptual framework for holonic manufacturing systems operation [34], work on the formation of holons and the feasibility of business process re-engineering methodologies for the creation of holonic manufacturing systems [35].

\section{GENERAL REPRESENTATION OF A SMALL ENTERPRISE}

SMEs are usually defined as companies with less than 500 employees. They can be considered as two distinct groups. The first group consists of the small manufacturing enterprises that are often owner-managed and occupy the demanding position of being at the end of the supply chain. On the one hand these can be small sophisticated businesses using high-tech equipment and on the other hand traditional businesses depending on high-quality craft skills and conventional manufacturing equipment. The management of such small companies is often very informal and based on an ad hoc approach, which relies heavily on the skills and knowledge of experienced employees. In the second group, represented by medium-sized manufacturing enterprises, the organizational management is somewhat different and more formal, but still distinctly different to the deterministic structures of larger enterprises.

In general, the organizational structure of SMEs can be simply viewed as a limited number of areas of activities. Each area of activity must have both its internal operations and relations with the rest of the business clearly identified. One of the simplest ways of describing the operation of SMEs is to classify its activities into three categories, as depicted in Fig. 1:
1. Executive activities. These include the supervision of daily operations, liaison with customers and suppliers, strategical planning, etc.

2. Business support activities. These consist in processing orders through a range of financial and production related activities. Typical activities are sales, marketing, costing, invoicing, inventory control, purchasing, process planning, routing and scheduling.

3. Manufacturing activities. These involve the implementation of production plans, monitoring of production processes and ensuring that the production orders are completed on time.

The planning and control requirements of both small and medium manufacturing enterprises are very similar in that they need high levels of agility and reactiveness to customer demands. These demands typically result from SMEs either being employed by larger companies as subcontractors to manufacture a small range of part types in a relatively predictable pattern, or processing the orders from a large number of smaller customers with a rich mix of part types and infrequent repeat orders. Therefore, the customer orders received by such SMEs can be viewed as:

(a) repeated orders of a limited number of part types or

(b) unpredictable orders of a wide range of part types.

Furthermore, an SME may choose to process such orders within different manufacturing areas. The repeated orders are usually processed in an advanced or more automated environment with less human intervention, designed to produce large batches of similar part types, whereas the unpredictable orders, which demand more flexibility, are mainly processed in human centred manufacturing areas, as shown in Fig. 2. The informal structure of such an enterprise provides an efficient and flexible working environment where operators can migrate from one manufacturing area to another, as and when required by the ever changing needs of the business.

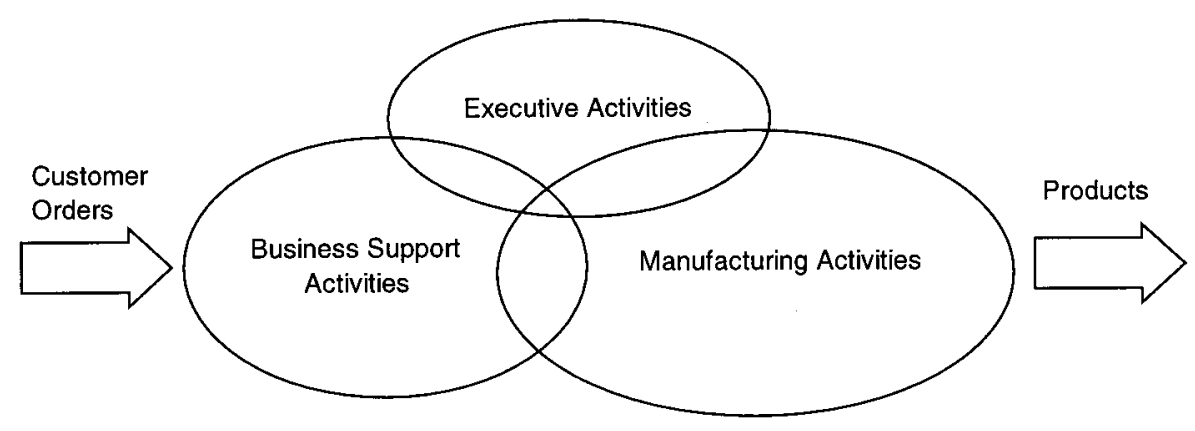

Fig. 1 Typical activities within an SME 


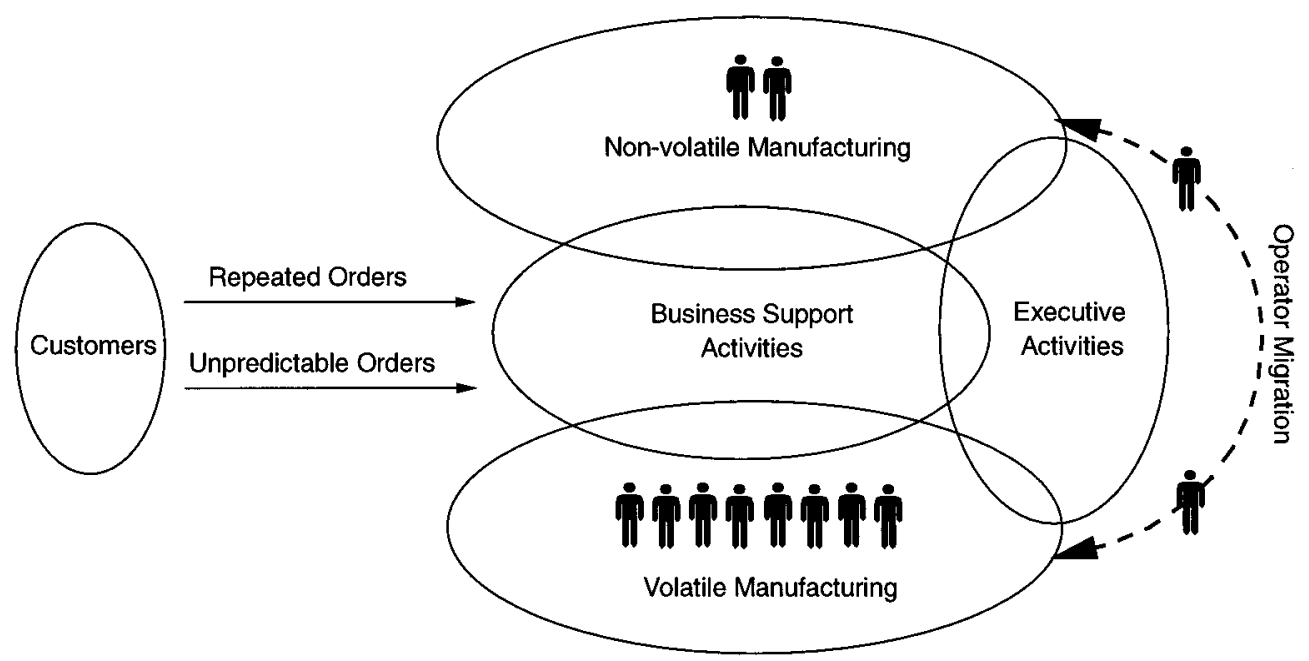

Fig. 2 General representation of the customer/SME relationship

\section{RESEARCH CONTEXT: THE REQUIREMENTS FOR OPERATIONAL PLANNING AND CONTROL OF SMES}

The fundamental assertions made in this paper are that the familiar information systems, production planning and control hierarchies and range of tools employed in larger companies are inappropriate for SMEs, and that utilization of appropriate IT tools significantly improves the manufacturing performance of SMEs. As a result, the initial efforts in this research have been directed towards identifying the specific information, integration and operational planning and control requirements of SMEs. This has been achieved through an extensive programme of industrial visits and formal interviews with employees, which has resulted in the identification of four major requirements most frequently highlighted by the key decision makers in such enterprises. These requirements are described below.

\subsection{Single order processing}

The overall lead time, i.e. the duration between receipt of customer orders and delivery of finished goods, can be divided into the order processing lead time and the production lead time, as shown in Fig. 3a. Order processing includes tasks such as finance, costing, invoicing, process planning, aggregate planning and material and resource requirements planning. Production processes can vary significantly in different manufacturing applications. In the case of a discrete part manufacturing company, these processes can include casting, metal cutting, forming, assembly, quality control, cleaning, decoration and packaging. The recent reviews of commonly adopted contemporary production planning systems have indicated that, within the majority of large manufacturing companies, the order processing lead time is often as long as (if not significantly longer than) the production lead time, as illustrated in Fig. 3b [7]. This is mainly due to the following:

1. The delays caused by rigid hierarchical planning structures within deterministic manufacturing companies often result in order processing activities being carried out by a large number of planners, often in various planning offices, at different times.

2. The complexity involved in processing orders in hierarchical PPC structures makes it impossible to deal with the orders individually. Therefore, the processing of customer orders is often delayed so that they can be accumulated over a period of time (which can be as long as a few days or even a week) and processed together to produce a master production schedule.

As the order processing lead time typically represents a significantly longer proportion of the overall lead time, compared with the production lead time, any improvement in these order processing activities could result in a major reduction in the total lead time. In comparison, optimization of production processing activities through increasing resources in manufacturing (e.g. purchase of new equipment, extra staff, overtime) can be costly and often does not result in a major reduction in overall lead time. This reduction is an even more important issue in SME applications, as orders often have a very short overall lead time and need to be rapidly processed to meet their due dates. Therefore, to be responsive to customer demand, any PPC structure adopted in an SME must attempt to minimize the order processing lead time, as indicated in Fig. 3c. To achieve this, there is a need to investigate novel production planning and control structures that rationalize and simplify the tasks involved in processing orders. In particular, such a novel PPC structure must incorporateprocedures that allow the customer orders to be processed individually when required, i.e. support single 


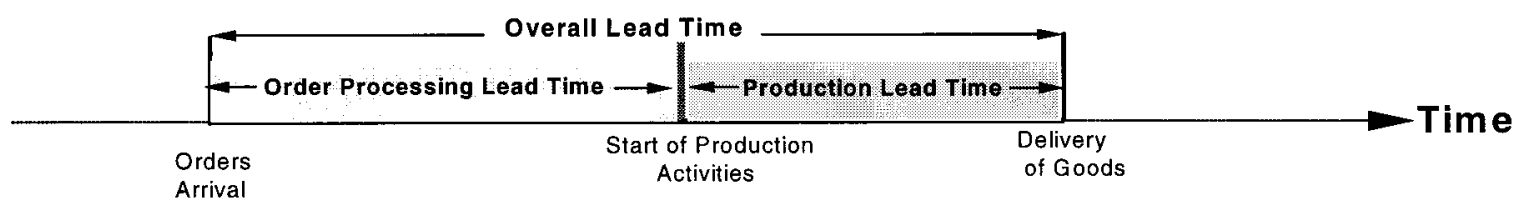

a) Definition of Lead Times for an Order in an Enterprise

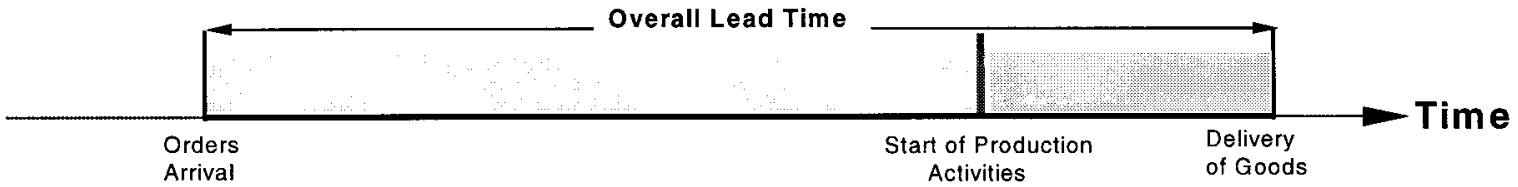

b) Division of Overall Lead Time in Large Enterprises

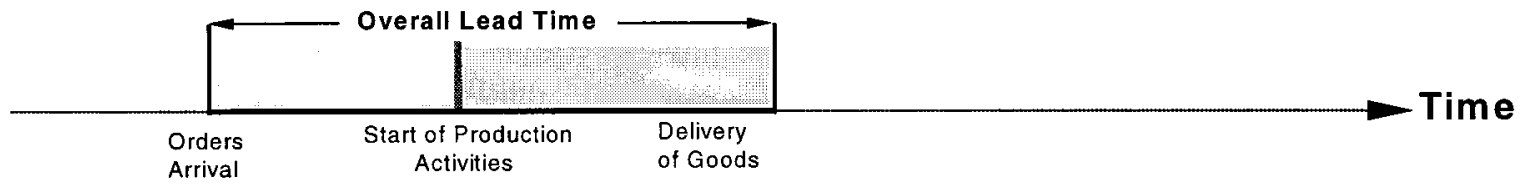

c) Division of Overall Lead Time Required by SMEs

Fig. 3 Division of the overall lead time into order processing and production lead times

order processing (SOP). It is clear that the simplification of order processing activities, and in particular the incorporation of SOP activities, results in the minimization of delays caused by the factors described above.

\subsection{Distributed decision support}

Planning, scheduling and control are problems of wellknown complexity, involving a wide range of decisionmaking activities. Traditionally, within more deterministic applications the planning and control decisions are often made centrally in a planning office, away from the production environment. This approach has two main disadvantages:

1. The latest shop-floor information and knowledge must be accessible to ensure the quality of the planning decisions.

2. The need to develop an appropriate scheduling tool that can effectively deal with the enormous complexity of the scheduling tasks owing to the existence of a wide range of constraints and competing manufacturing goals.

These disadvantages, in one respect, underpin the necessity for a comprehensive information network that links the planning and production activities, but also highlight the inefficiency of centralized production planning and control in meeting the challenges of modern manufacturing applications [36, 37]. In SME applications, production processes are often carried out by a range of multiskilled operators grouped into production

B05698 (C) IMechE 1999 teams, who are responsible for a range of activities and work in an autonomous manner. Therefore, a new decision-making structure is required to allow local planning and control decisions to be made by teams of production operators throughout a manufacturing SME, and in particular within its shop-floor environment. Obviously, such a distributed decision-making structure must be supported by appropriate rule sets and IT tools to avoid production anarchy.

\subsection{Interactive planning}

In larger, more deterministic manufacturing applications supported by hierarchical PPC structures, planning and control functions are well defined and often carried out by a number of specific planners such as the material requirements planner, process planner, scheduler and shop-floor controller. Access to the planning and control system is restricted and is made available only to these production planners. Production operators often receive generated work plans (work-to-lists) in the form of a printed copy of the schedule and often do not have direct access to the planning system. However, as discussed in Section 4.2, to incorporate a distributed decision-making structure, any planning and control structure specifically designed for SMEs must support frequent interactions not only with production planners but also with the production operators. At the same time, such an interactive planning and control structure should be supported by suitable security measures to provide appropriate levels of functionality and 
responsibility to the planners and operators in terms of making changes to generated manufacturing plans. It should be noted that interactive capabilities must be designed in such a way that they are easy and relatively quick to use, otherwise they would not be practical for use within the shop-floor environment.

\subsection{Reactive planning}

Planning and scheduling rules can be classified as static or dynamic. Static rules have performance indices that are independent of time, whereas dynamic rules are time dependent and must therefore be used in conjunction with real-time data. Static rules are used within a 'predictive production planning and control' structure, using a technique commonly referred to as off-line planning and control. However, dynamic scheduling rules are used within a 'reactive production planning and control' structure which is often referred to as 'real-time' or 'on-line' planning and control $[38,39]$. Within a real-time planning and control system there is a need for a detailed model of the manufacturing system that has been integrated via a computer network into the physical resources (production system) on the shop-floor. This model continuously receives information on the progress of previous production instructions and the system status through shop-floor data collection facilities. The updated information is then used to generate further production instructions and to make appropriate corrective modifications to overcome any possible problems caused by manufacturing disturbances. The success of such systems relies very much on the design of the information system and the quality of data being exchanged between the PPC system and the shop-floor. SMEs are required to be extremely agile in response to customer pressure and therefore it is apparent that any proposed solution for the production planning and control problems of SMEs must be reactive and based on a dynamic real-time approach.

\section{HOLONIC STRUCTURE FOR PLANNING AND CONTROL OF SMES}

In order to develop a planning and control structure that satisfies the requirements outlined in Section 4, an initial model has been defined which considers the business as a complete entity and proposes new modelling methodologies based on the use of holonic ideas. In this model, a holon is defined as an autonomous, cooperating agent consisting of an information or material processing subsystem and one or more humans who may or may not be present at any given time, as shown in Fig. 4.

SMEs can be viewed as an inherently holonic enterprise where the human operators interact in a manner consistent with the rules and practices of the business, often with the minimum of information support [18]. These operators usually employ manufacturing equipment that is often disconnected in terms of information flow. The shortcomings associated with the current practices in the utilization of IT tools in small enterprises are as follows:

1. There is a lack of a continuous information network being used between executive, business and manufacturing activities.

2. The DNC network supports the bidirectional messaging between the dominant node and the individual workstation nodes, but not between the various workstation nodes.

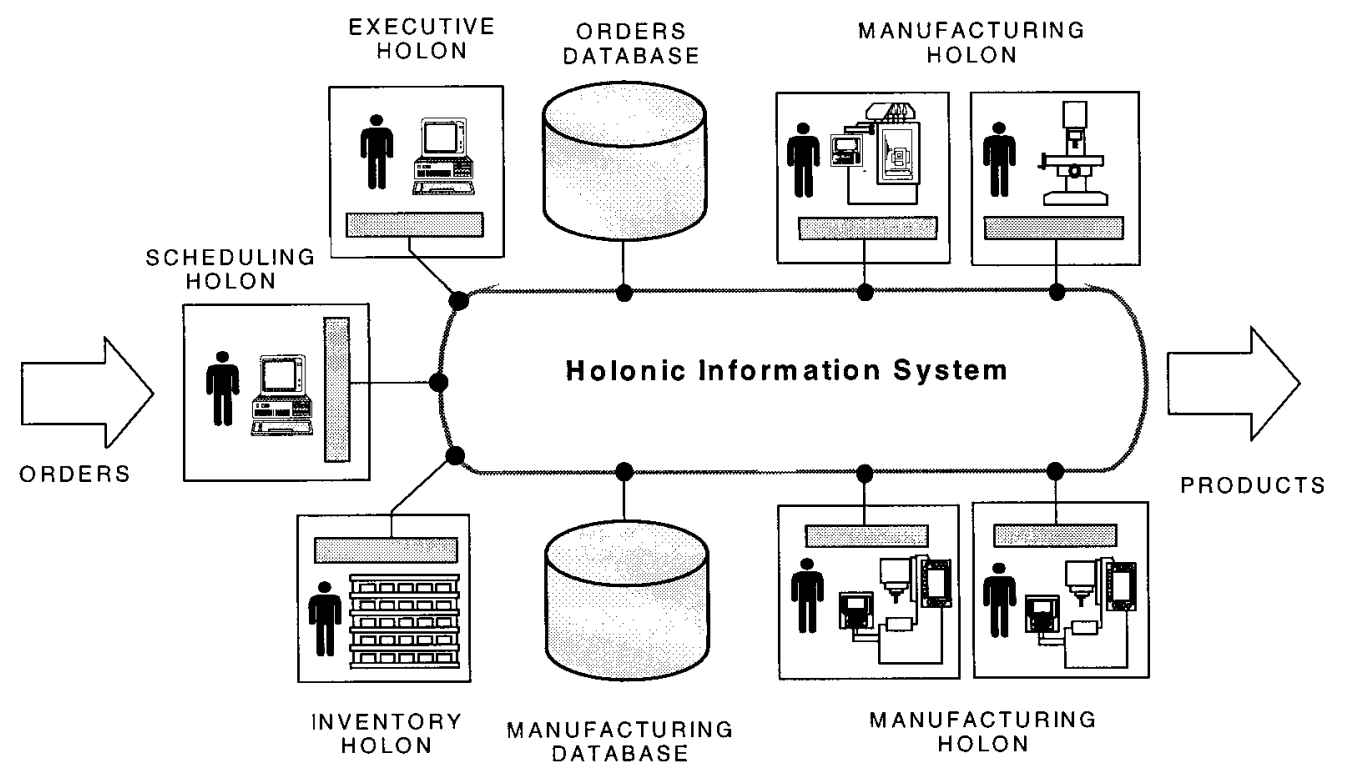

Fig. 4 Holonic structure for planning and control of SMEs 
3. The SME is often supported by IT tools that provide little effort to support human centred activities.

The design and implementation of the appropriate information system for the application of human centred manufacturing is described elsewhere $[\mathbf{4 0}, \mathbf{4 1}]$. The goal of such an information system specification and design is to reduce all the information subsystems into one homogeneous integrated system. The achievement of this goal implies that all the rules governing the operation of the individual activities within the business are known and correctly implemented in a total enterprise information network (see Fig. 4), thus providing powerful support to the daily operation of an enterprise. Such an information system has to provide the information support in a way that enhances the collaborative activity within the business and strengthens the role of the individual. The implementation of such an information network is seen as of paramount importance in the realization of a holonic planning and control structure. Therefore, one of the major objectives of the research has been to provide a range of enhanced organization holons in which human operators and manufacturing workstations are paired to share the same interface and are both integrated into an information network supported by specially designed IT tools, as shown in Fig. 4 , referred to as the 'holonic information system' (HIS). The HIS network is supported by a manufacturing and an order database. Furthermore, based on this information infrastructure, a holonic planning and control structure tailored to the needs of SMEs has been generated which consists of an inventory and a scheduling holon. The remaining sections of this paper describe the principle, design and implementation of this novel planning and control structure.

\section{DISTRIBUTED AUTONOMOUS REAL-TIME PLANNING AND CONTROL}

The manufacturing operations of small enterprises are mainly typified by the application of human centred manufacturing. Such enterprises are required to be extremely agile in response to customer pressure and normally rely on meeting these demands by the use of considerable ingenuity and flexibility. In order to design a planning and control solution tailored to the needs of SMEs, based on the requirements described in Section 4 and the holonic structures described in Section 5, there is potential to explore a novel real-time planning and control approach. This approach is referred to as 'distributed autonomous real-time' (DART) planning and control which aims to:

(a) reinforce the autonomous and cooperative attributes of individual operators within production planning and control tasks of small manufacturing enterprises through the implementation of a distributed decision-making network supported by an appropriate holonic information infrastructure;

(b) provide structures for the traditional influence of the manufacturing controller (e.g. the business owner, production manager) in the form of infrequent interventions to reprioritize orders and allocate jobs to certain resources as and when required.

Based on the holonic model described above and illustrated in Fig. 4, the main tasks and the sequence in which they are carried out within the DART approach is illustrated in Fig. 5. These tasks are:

1. Order confirmation. Small subcontracting enterprises usually receive a large number of widely varying customer orders. These orders must be initially handled to ensure that manufacturing capabilities required to fulfil the orders are supported by the production system, and that the orders can be completed within the expected cost indices and delivery dates. This task is carried out through a joint effort by the executive holon, inventory holon and scheduling holon.

2. Process planning. Manufacturing operations, operations sequences, operation times and the resources required (workstations, tools, fixtures) to process an order must be identified, and based on this information a process route through the production system is developed by the scheduling holon. Such information is then stored in an order or a product database.

3. Inventory control. The availability of raw material, tools, fixtures and any other resource required is considered by the inventory holon and, where necessary, appropriate purchasing instructions are generated.

4. Generation and maintenance of production order list (POL). In this novel approach it is assumed that a list of all production orders is maintained and that this list can be updated in real time, as and when required. Furthermore, access to the POL is made available both to planners and operators (i.e. all the holons in the system) via an information network similar to that described in Section 5. Based on information generated by process planning and inventory control tasks, a priority and due date are assigned to an order by the scheduling holon. Such a production order is then added to the POL (on a real-time basis).

5. Selection/allocation of a production order. The technique used to carry out this task distinguishes DART from the other reactive planning and control approaches. In a centralized reactive approach a production order is allocated (pushed) to a workstation by a production planner upon completion of another order. In the distributed reactive planning 


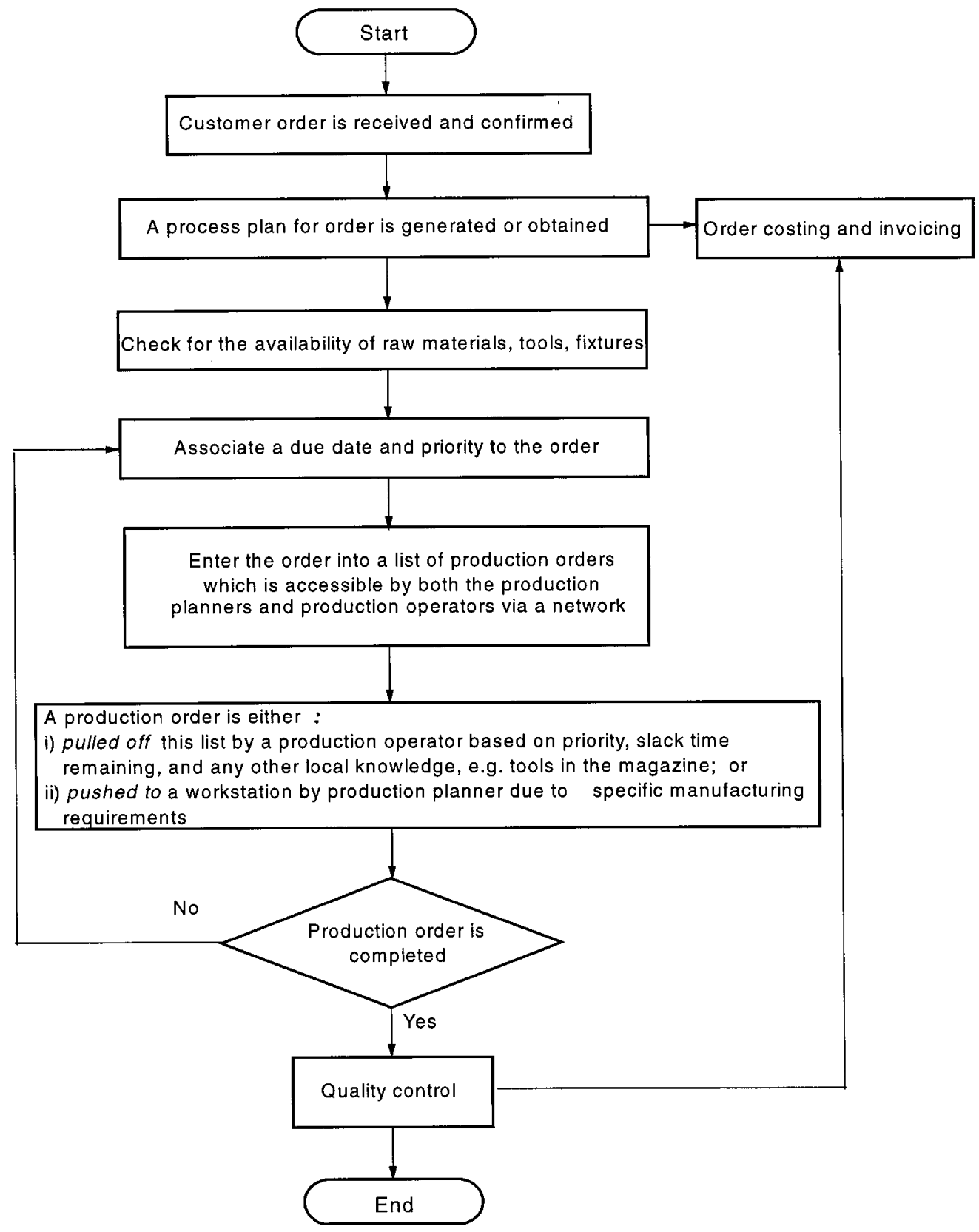

Fig. 5 Main tasks involved in the processing of orders within the DART-based PPC

and control approach, the various sections of the total production facility (e.g. manufacturing zones, teams, cells) select (pull) a range of appropriate production orders upon completion of their previous orders, using a job bidding algorithm [42]. In the DART approach, in order to incorporate localized knowledge in planning decisions the utilization of distributed reactive techniques is strongly encouraged, and therefore in the normal mode of operation a job is selected by the operator of an individual manufacturing holon. This job is then flagged in the POL to be allocated to that manufacturing holon. However, there are complementary structures that allow a particular production order to be forced upon a manufacturing holon, owing to specific requirements, by the executive or scheduling holons.

6. Order completion reporting. The completion of a production order is also flagged in the POL and a range of appropriate reports on materials, tools, fixtures and machine set-ups used is generated. Furthermore, additional information that might be used to improve the processing of similar production orders is recorded in the order and manufacturing databases. In the case of any manufacturing disturbances (e.g. machine breakdown, shortage of raw material) the production planner (i.e. the scheduling holon) is informed via the information network, 
using the messaging facilities, so that the work can be redirected or a new due date can be assigned to the order.

A number of hierarchical reference models have been developed for the overall planning and control of a modern manufacturing system [43]. These hierarchical architectures are closely related to the structure of traditional centralized planning and control systems and are characterized by a strong master-slave relationship. Such architectures often propose a hierarchy consisting of four or five control levels such as factory, shop, cell and equipment. However, within small manufacturing enterprises, in particular the small metalworking enterprises considered in this paper, with a limited production facility such hierarchical reference models consisting of four or five levels could not be supported.

In such applications the heterarchical systems provide an attractive alternative architecture in which a collection of loosely coupled autonomous subsystems are cooperating to achieve an overall manufacturing goal. Therefore, in order further to define the order processing tasks described above, a simpler and flatter view of SME organizations has been adopted which classifies these planning and control tasks into two categories of factory level and workstation level, as illustrated in Fig. 6. Factory level tasks include the executive and business support activities carried out by an executive holon, inventory holon and scheduling holon and workstation level tasks represent the manufacturing activities undertaken by manufacturing holons.
The factory level tasks convert the customer orders into a set of production orders and the workstation level tasks execute these production orders to generate the required products. Customer orders for the metalworking SMEs in the engineering-to-order sector can take the form of:

(a) an order for a new part type that requires a process planning activity (manual or computerized process planning);

(b) an order for a part type previously manufactured, in which case the process planning information is stored in the order database;

(c) an order for an established part type that is produced regularly as part of a company's product range, in which case the process planning information for such part types are often kept with the product database.

The generation/retrieval of process planning information by the scheduling holon leads to inventory control activities which in turn allows orders with appropriate priorities and due dates to be added to the POL. At this stage, the planner within the scheduling holon can take the decision of forcing the production order on to a specific manufacturing holon or allow it to be selected and processed as and when appropriate by one of the manufacturing holons. At workstation level, a large number of criteria, such as priorities, due dates, slack times and commonality with present machine and tool set-ups, are taken into account to select and process an order. The completion of the production order is then

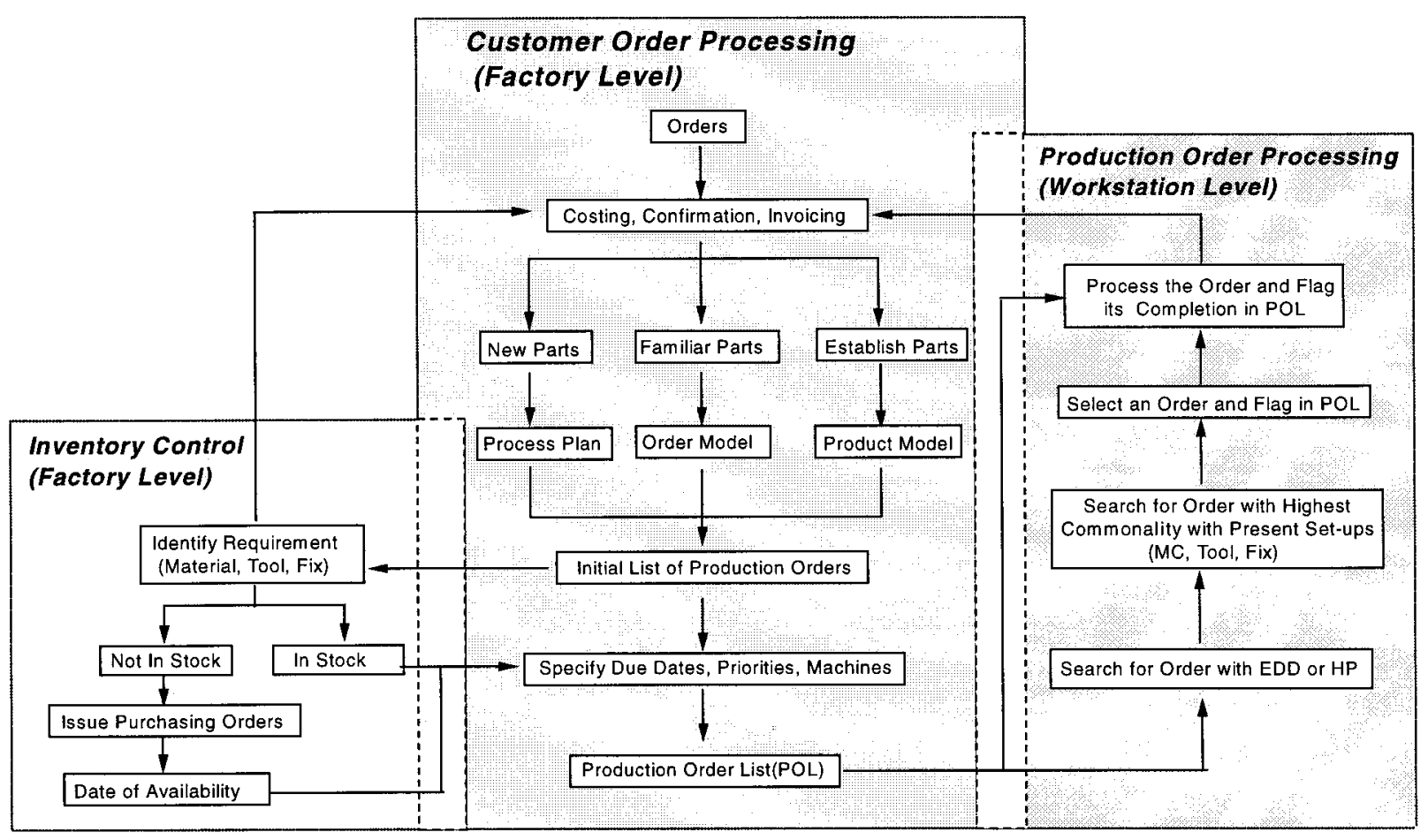

Fig. 6 Factory and workstation level tasks involved in the processing of orders within the DART-based PPC 


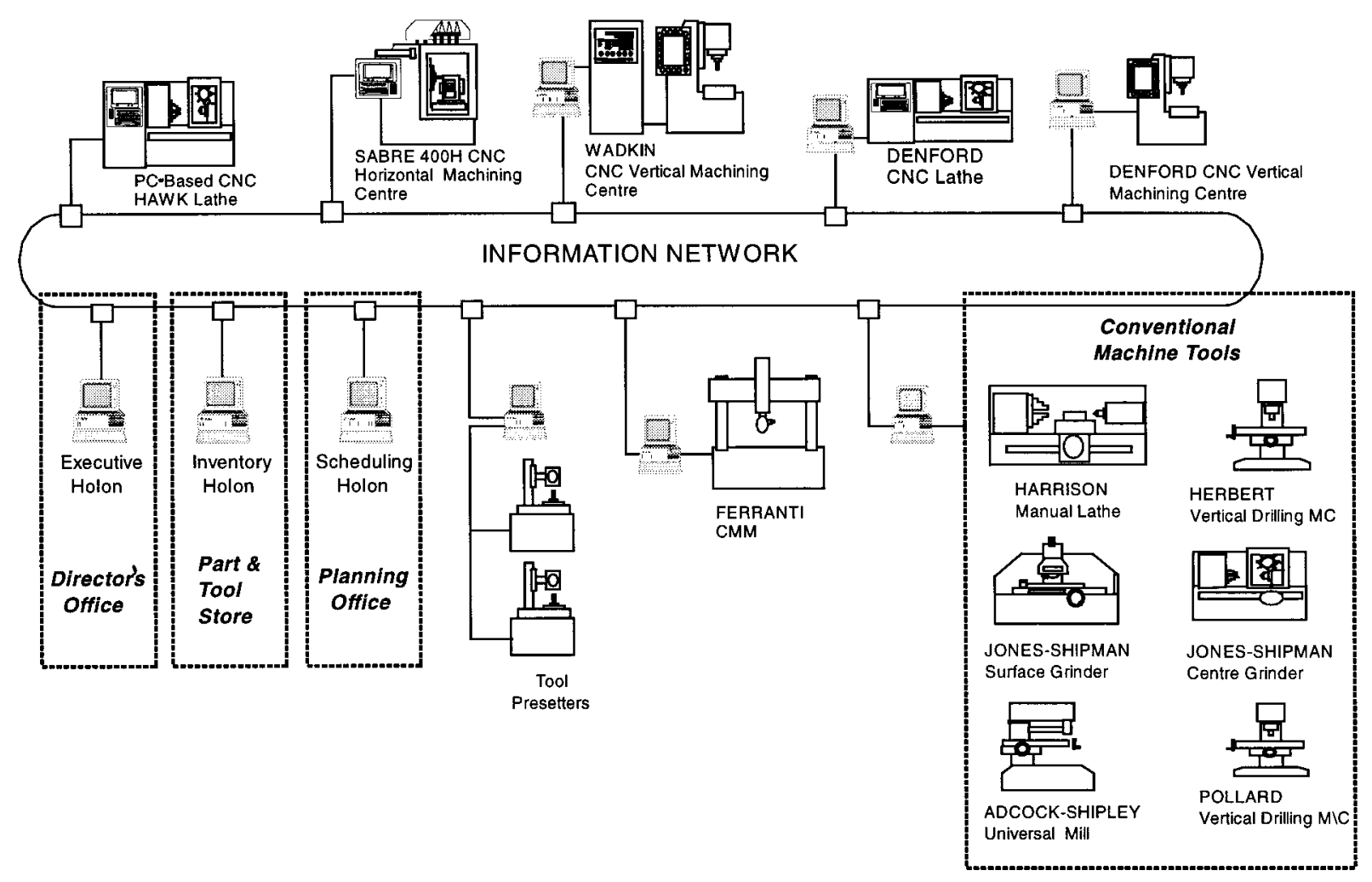

Fig. 7 Schematic of typical production facilities within a small metalworking SME

flagged and appropriate information with regard to processing the order is recorded.

\section{APPLICATION OF DART WITHIN A SMALL METALWORKING SME}

There are a large number of tasks such as process planning, inventory control, aggregate planning, resource allocation and production control that are involved in the processing of customer orders. Although the majority of these tasks need to be carried out in some format within any manufacturing application, the sequence and techniques used to carry out such tasks very much depend on the specific application. In order to illustrate the principles of the DART planning and control structure, an application of a typical small metalworking enterprise has been adopted. Such a company, typically within the engineering-to-order sector, is used mainly as a subcontractor to machine a wide range of part types in small to medium batch sizes. A typical configuration for the production facility of such a metalworking enterprise is illustrated in Fig. 7.

This production facility consists of a number of computer numerical control (CNC) milling and turning machines, $\mathrm{CNC}$ vertical and horizontal machining centres, a range of conventional machine tools, a coordinate measuring machine (CMM) and a number of tool presetting workstations. The manufacturing facilities are linked together via a computer network (in this example a Microsoft network) and each area of manufacturing activity (a manufacturing holon) is equipped with a computer (running the Windows NT operating system). Each of these manufacturing holons is manned by one or more operators. It should be noted that such manufacturing holons can consist of a single workstation (e.g. a PC-based CNC machining centre) or a collection of workstations grouped together around a computer node linked to the information network (e.g. a number of conventional machine tools or tool presetters). In addition, a number of other computers within the planning office, part and tool store and managing director's office are linked to this information network, representing the scheduling holon, inventory holon and executive holon.

A special-purpose user interface has been designed and implemented which is hosted by every computer linked to the network. This user interface not only provides access to all the software modules supported by the information network, namely the order database, the manufacturing database, the inventory module and the DART scheduler, but also enables the operators of various holons to execute a range of special-purpose software packages that are required to support their daily functionality. For example, the tool presetter holon can run a tool management system which stores the tool assembly information and presetting measurements, the CMM holon uses a CAD 
system to obtain the part measurements in design drawing to be compared with actual measurements, and the machining holons can make minor changes to the machining codes using a special-purpose editor. In addition, this user interface supports a range of bidirectional messaging activities between the various holons within the system, so that not only the instructions can be downloaded by the executive holon or planning holon to the manufacturing holons but also various types of queries (negotiations) can be made between each holon in the system.

\subsection{Computational viewpoint of DART}

A commercial finite capacity planning system, called PREACTOR [44], has been used as the basic tool for the development of a prototype DART system. PREACTOR is a highly configurable planning system which utilizes graphical user interfaces for ease of use and rapid access to information. It has a modular structure of functionality, named accordingly PREACTOR 200, 300, 400 and 500, starting at its lowest level of functionality with PREACTOR 200 which supports a limited number of the most common scheduling algorithms. In PREACTOR 300 this list of scheduling rules is expanded. PREACTOR 400 and 500 allow user defined rules to be implemented (using visual basic pro- grammes) and enable schedules to be generated on the basis of a combination of such rules (i.e. multicriterion scheduling).

In addition, all PREACTOR modules provide the users with a data definition language to develop customized database structures. The PREACTOR database has a relational structure in which data elements are stored in a number of tables. The tables appear as a set of computer screens, divided into rows and columns (see Fig. 8). Furthermore, each row is connected to a dialogue screen that includes a number of data fields. The manufacturing information is entered into the database by typing the various data values in these fields, as shown in Fig. 9. Figure 8 shows an example of a POL and Fig. 9 illustrates the associated dialogue screens used to input the relevant information to this list.

The prototype DART planning and control system consists of a number of PREACTOR modules that have been significantly reconfigured and enhanced to generate a scheduling holon, together with a number of planning modules that are hosted by all the other holons within the system. These planning modules enable other holons (i.e. executive holon, inventory holon and manufacturing holon) to have access to the POL and other functional modules provided by the scheduling holon. The access to the scheduling holon has been

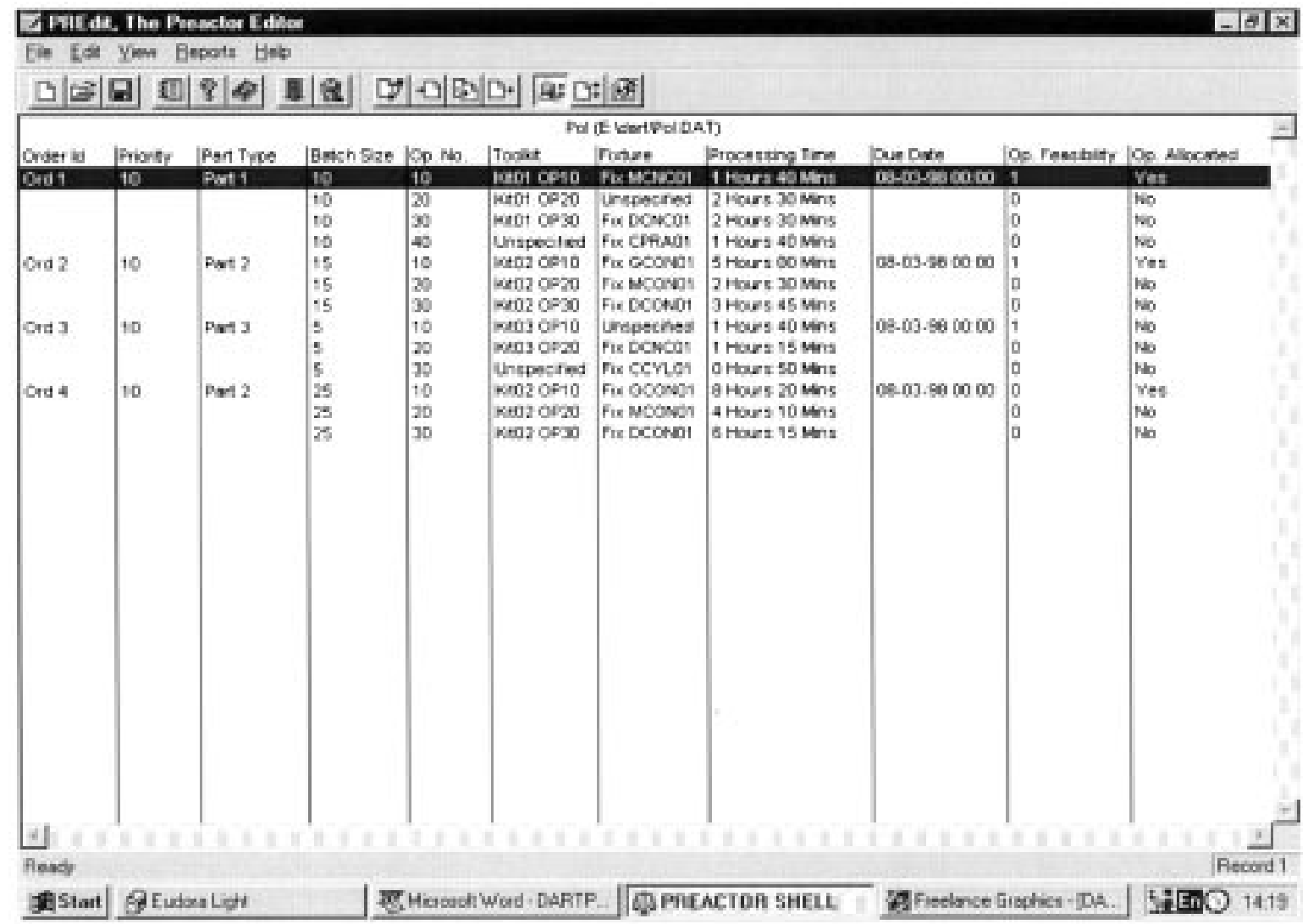

Fig. 8 Production order list (POL) of the DART scheduler 


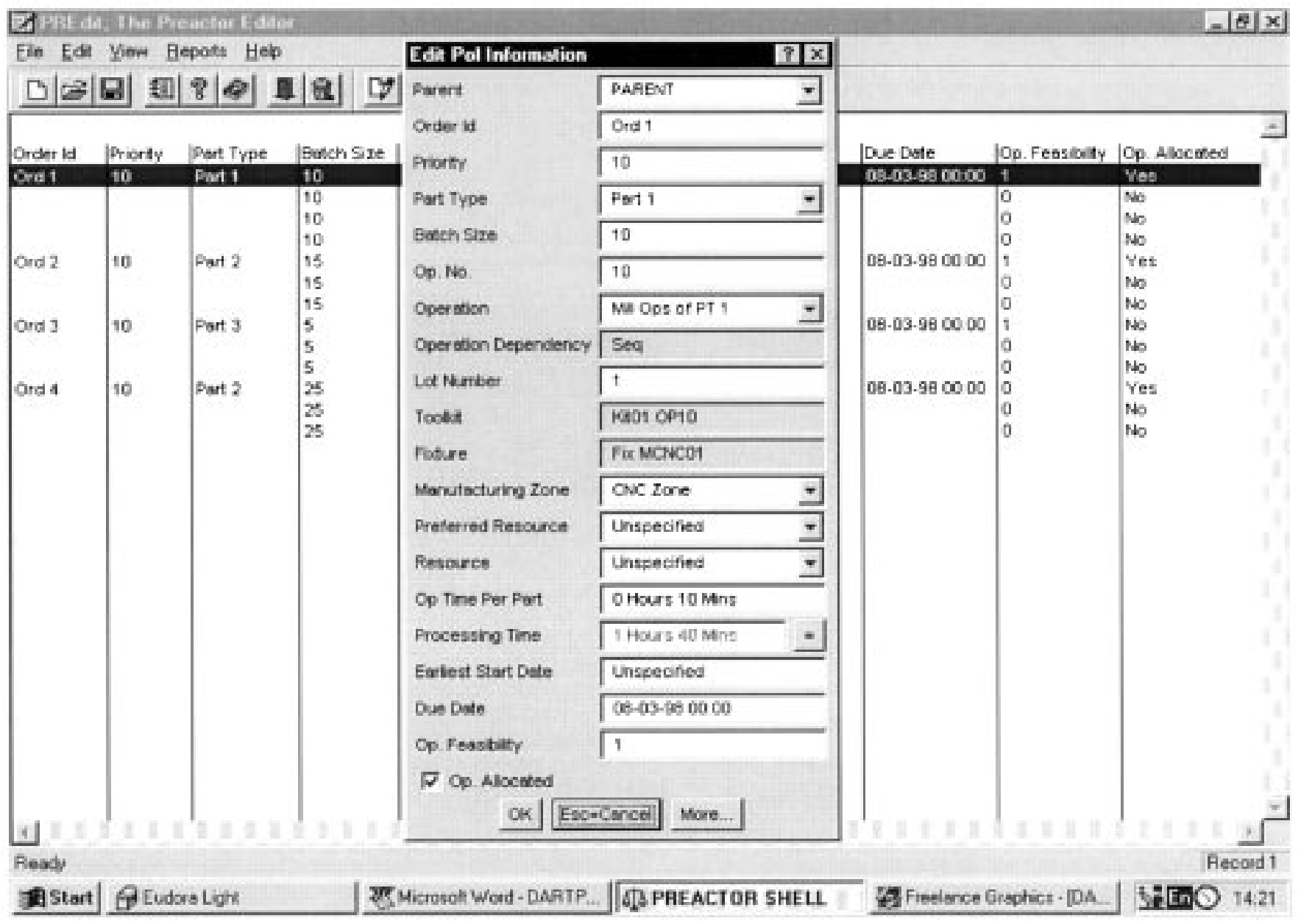

Fig. 9 Information stored in the production order list (POL) of the DART scheduler

organized on the basis of a number of security levels which limits the functional capabilities of these planning modules, depending on the host holon. For example, a manufacturing holon can access POL to view and select a production order but cannot reprioritize, or change the due date of an individual production order without prior negotiations with the scheduling holon. Similarly, manufacturing holons can access data on part operations, tooling and fixture requirements to obtain and update information, but, for example, for obvious reasons they would not be able to update information related to the list of resources with the production system.

An example set of software modules utilized in the various holons within the described production system (see Fig. 7) is illustrated in Fig. 10. These include:

(a) the use of a graphical editor to provide a visual representation of current schedules within an executive holon;

(b) the utilization of a planning module to access the POL and flag the completion of a production order within the PC-based CNC turning holon; (c) the use of a CAD/CAM system to carry out minor amendments to the generated machining code for a part type on the CNC vertical machining holon; and finally

(d) the list of selected jobs (i.e. production orders) for the conventional manual machining holon, together with their tool and fixturing requirements and the required due dates.

Clearly, the utilization of such a planning and control system within SMEs satisfies the major requirements outlined in Section 4 in that it allows orders to be processed individually as soon as the customers have confirmed; it supports a distributed decision-making network where the majority of planning decisions are made by the appropriate employees who have access to the latest information on system status; it provides an interactive planning system where a range of managers, planners and operators can interact with the planning and control system; and finally it incorporates a reactive real-time planning and control approach that provides the agility and flexibility required to meet the customer demands within SME applications. 


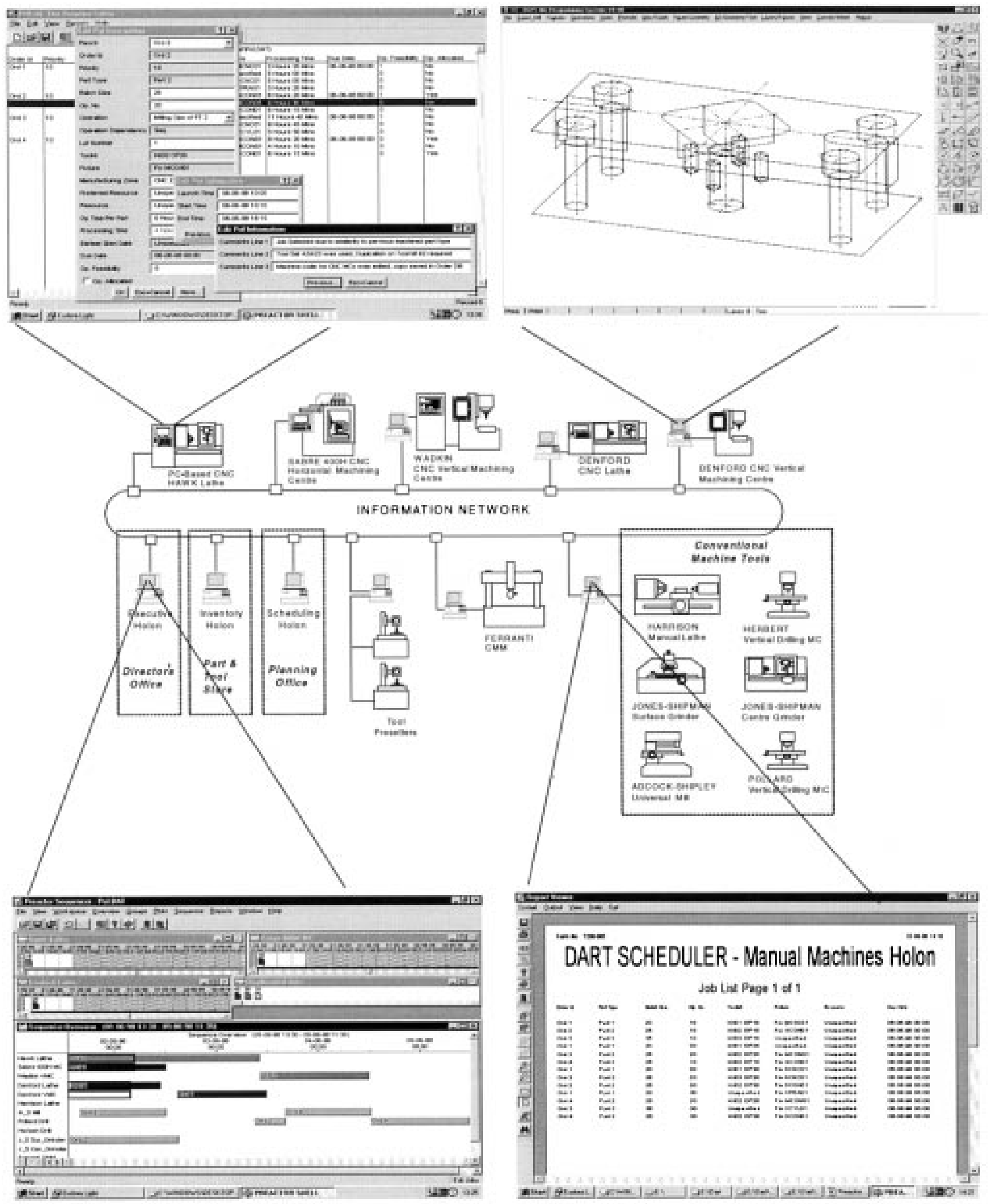

Fig. 10 Typical set of software modules used by various holons 


\section{CONCLUSIONS}

The fundamental assertion made in this paper is that the familiar information systems, production planning and control hierarchies and range of tools employed in larger companies are inappropriate for SMEs. It has also been established that an effective production planning and control structure tailored for the needs of such enterprises must:

(a) reduce the delays in processing the customer orders within pre-production activities,

(b) support distributed decision making to strengthen the role of production operators,

(c) support frequent interactions with both the production planners and operators,

(d) incorporate a dynamic real-time approach to enable extreme agility in responding to customer pressure.

Based on these requirements and the use of holonic manufacturing concepts, a distributed autonomous real-time planning and control structure has been proposed which reinforces the autonomous and cooperative attributes within production planning and control tasks of small manufacturing enterprises. It has been argued that the success of such an approach requires a simpler view of an SME organization with a flatter management, planning and control structure. As an example, a semi-heterarchical structure with two categories of factory level and workstation level activities has been defined. Factory level tasks include the executive and business support activities and workstation level tasks that represent the manufacturing activities. The factory level tasks convert the customer orders into a set of production orders and the workstation level tasks execute these production orders to generate the required products. It should also be emphasized that realization of a DART-based production planning and control system is very much dependent on the design and implementation of a holonic information system for the application of human centred manufacturing to provide the information support in a way that enhances collaborative activity within the business and strengthens the role of the individual. Finally, it is believed that the implementation of a DART system provides the agility and responsiveness required by SMEs to stay competitive and meet the volatile challenges of modern manufacturing applications.

\section{ACKNOWLEDGEMENTS}

This work has been carried out as part of a government funded research programme from the Control, Design and Production Group of the Engineering Physical
Science Research Council, entitled 'IT Tools to Improve the Manufacturing Performance of Metalworking SMEs' (GR/L27077). The authors would like to acknowledge the work of the researchers in the Advanced Manufacturing Technology Centre at Loughborough University and the supporting work of the industrial collaborators: The CIMulation Centre Limited, ISIS Informatics Limited, Camtek Limited, Plenty Limited, A.O. Henton Limited and Quadro Engineering Limited.

\section{REFERENCES}

1 Suda, H. Future factory system formulated in Japan. Jap. J. Advd Automn Technol., 1990, 23(3), 51-61.

2 Okino, N. Bionic manufacturing systems - bio-model on based systems design. In Proceedings of CAM-I Annual International Conference, New Orleans, Louisiana, 1989, pp. 485-492.

3 Warnecke, H. J. The Fractal Company: a Revolution in Corporate Culture, 1993 (Springer-Verlag, UK).

4 Tharumarajah, A., Wells, A. J. and Nemes, L. Comparison of the bionic, fractal and holonic manufacturing systems concepts. Int. J. Computer Integrated Mfg, 1996, 9(3), 217-226.

5 Halsall, D. N., Muhlemann, A. P. and Price, D. H. R. A review of production planning and scheduling in smaller manufacturing companies in the UK. J. Prod. Plann. and Control, 1994, 5(5), 485-493.

6 Karatsu, H. Management principle of small manufacturing firms in Japan. In Proceedings of 3rd International Conference on Manufacturing Technology, Hong Kong, 1995, pp. 20-22.

7 Sridharan, V. and Kanet, J. J. Production planning and control systems - state of the art and new directions. In Information Management in Computer Integrated Manufacturing (Eds H. H. Adelsberger et al.), 1995, pp. 195216 (Springer-Verlag, Berlin).

8 Veeramani, D., Bhargava, B. and Barash, M. M. Information system architecture for heterarchical control of large FMSs. J. Computer Integrated Mfg Syst., 1993, 6(2), 76-92.

9 Chan, F. T. S. and Tang, N. K. H. Optimising production scheduling in small scale industries by using PC-simulation package. In Proceedings of 3rd International Conference on Computer Integrated Manufacturing, 1995, Vol. 2, pp. 869-876.

10 McKay, K. N., Safayeni, F. R. and Buzacott, J. A. A review of hierarchical production planning and its applicability for modern manufacturing. J. Prod. Plann. and Control, 1995, 6(5), 384-394.

11 Koestler, A. The GHOST in the MACHINE, 1967 (Arkana Books, London).

12 Leeuwen, E. H. and Norrie, D. Holons and holarchies. Mfg Engng, 1997, 76(2), 86-88. 
13 Groumpos, P. P. The challenges of intelligent manufacturing systems (IMS): the European IMS information event. J. Intelligent $M f g, 1995,6(1), 67-77$.

14 Matthews, J. Organisational foundations of intelligent manufacturing systems - the holonic viewpoint. Computer Integrated Mfg Syst., 1995, 8(4), 237-243.

15 Winkler, M. and Mey, M. Holonic manufacturing systems. Eur. Prod. Engng, 1994, 18(3-4), 10-12.

16 Nakane, J. and Hall, R. W. Holonic manufacturing: flexibility - the competitive battle in the 1990s. Prod. Plann. and Control, 1991, 2(1), 2-13.

17 Christensen, J. Holonic approach compared to other common concepts. In Proceedings of 1st European Conference on Holonic Manufacturing Systems, Hannover, 1994.

18 Mezgar, I. and Kovacs, G. L. Co-ordination of SME production through a co-operative network. J. Intelligent Mfg, 1998, 9, 167-172.

19 Van Brussel, H., Valckenaers, P., Wyns, J., Bongaerts, L. and Detand, J. Holonic manufacturing systems and LiM. In IT and Manufacturing Partnerships (Eds J. Browne et al.), 1996, pp. 185-196.

20 Markus, A., Kis Vancza, T. and Monostori, L. A market approach to holonic manufacturing. Ann. CIRP, 1996, 45(1), 433-436.

21 Sugimura, N. and Moriwaki, T. Modelling of holonic manufacturing system and its application to real-time scheduling. Proc. CIRP Semin.—Mfg Syst., 1996, 25(4), 345-352.

22 Sousa, P. and Ramos, C. A dynamic scheduling holon for manufacturing orders. J. Intelligent $\mathrm{Mfg}, 1998$, 9, 107112.

23 Cselenyi, J. and Toth, T. Some questions of logistics in the case of holonic production systems. J. Intelligent $\mathrm{Mfg}$, 1998, 9, 113-118.

24 Tonshoff, H. K. and Winkler, M. Shop control for holonic manufacturing systems. Proc. CIRP Semin.-Mfg Syst., 1996, 25(3), 227-281.

25 Overmars, A. H. and Toncich, D. J. Hybrid FMS control architectures based on holonic principles. Int. J. Flexible Mfg Syst., 1996, 8(3), 263-278.

26 Bengoa, A., Gluch, S. and Jacobs, H.-J. An approach to holonic components in control of machine tools. Ann. CIRP, 1996, 45(1), 437-440.

27 Tanaya, P. I., Detand, J. and Kruth, J.-P. Holonic machine controller: a study and implementation of holonic behaviour to current NC controller. Computers in Industry, 1997, 33(2-3), 323-333.

28 Van Brussel, H., Valckenaers, P., Bongaerts, L. and Wyns, J. Architectural and systems design issues in holonic manufacturing systems. In Proceedings of 3rd IFAC/ IFIP/IFORS Workshop on Intelligent Manufacturing Systems IMS'95, 1995, pp. 1-6.

29 Bongaerts, L., Wyns, J., Detand, J., Van Brussel, H. and Valckenaers, P. Identification of manufacturing holons. In Proceedings of European Workshop on Agent-Oriented Systems in Manufacturing, Berlin, Germany, 1996.
30 Bongaerts, L., Valckenaers, P., Van Brussel, H. and Wyns, J. Schedule execution for a holonic shop floor control system. In Proceedings of Advanced Summer Institute (SAI) 95 of the N.O.E. on Intelligent Control of Integrated Manufacturing Systems, Lisbon, Portugal, 1995.

31 Wyns, J., Van Brussel, H., Valckenaers, P. and Bongaerts, L. Workstation architecture in holonic manufacturing systems. In Proceedings of 28th CIRP International Seminar on Manufacturing Systems, Johannesburg, South Africa, 1996.

32 Kilic, S.E. and Saygin, C. On changing manufacturing paradigms from strict hierarchies to holonic organisations. In Proceedings of 1st Working Group Meeting, Ankara, Turkey, 1996, pp. 1-14.

33 Kayaligil, S. Initial thoughts on holon formation in TAKSAN. In Proceedings of 1st Working Group Meeting, Ankara, Turkey, 1996, pp. 38-46.

34 Gullu, R. and Ozdemirel, N.E. Initial thoughts on HMS operation. In Proceedings of 1st Working Group Meeting, Ankara, Turkey, 1996, pp. 31-37.

35 Hoffman, Z. Holonic systems and business process reengineering. In Proceedings of 1st Working Group Meeting, Ankara, Turkey, 1996, pp. 47-63.

36 Ramaswamy, S.E. and Joshi, S. Distributed control of automated manufacturing systems. Proc. CIRP Semin.Mfg Syst., 1996, 25(4), 353-359.

37 Kokkinaki, A.I. and Valavanis, K.P. A distributed task planning system for computer-integrated manufacturing systems. J. Intelligent Mfg, 1996, 7(4), 293-309.

38 Hadavi, K., Hsu, W.-L., Chen, T. and Lee, C.-N. An architecture for real time distributed scheduling. In Artificial Intelligence Applications in Manufacturing (Eds A. Famili et al.), 1992, pp. 215-234 (AAAI Press, Cambridge, USA).

39 Sohier, C. and Bourdet, P. Real time scheduling of a production cell based on a multi-agent system. In Proceedings of International Conference on Industrial Engineering and Production Management, Mons, Belgium, 1993, pp. 931-939.

40 Deen, S. M. Cooperation issues in holonic manufacturing systems. Information Infrastructure Systems for Manufacturing, 1993, pp. 401-412 (Elsevier Science B.V./NorthHolland).

41 Toh, K. T. K., Newman, S. T. and Bell, R. An information system architecture for small metal-working companies. Proc. Instn Mech. Engrs, Part B, Journal of Engineering Manufacture, 1998, 212(B2), 87-103.

42 Chandar, J. and Talavage, J. Intelligent dispatching for flexible manufacturing. Int. J. Prod. Res., 1991, 29(11), 2259-2278.

43 Xiang, D. and O'Brien, C. Cell control research-current status and development trends. Int. J. Prod. Res., 1995, 33(8), 2325-2352.

44 The CIMulation Centre. The PREACTOR User Manuals, 1994 (The CIMulation Centre, Chippenham). 